\title{
DETERMINANTS OF SURGICAL SITE INFECTION IN RURAL KANPUR, INDIA
}

Hariom Sharan, Aditya Prakash Misra, Ritu Mishra.

1. Assistant Professor, Department of Microbiology. Rama Medical College, Hospital and research centre, Kanpur, UP.

2. Associate Professor, Department of Radiodiagnosis. Rama Medical College, Hospital and research centre, Kanpur, UP.

3. Assistant Professor, Department of OBG. Rama Medical College, Hospital and research centre, Kanpur, UP.

\section{CORRESPONDING AUTHOR}

Dr. Hariom Sharan

Assistant Professor, Dept. of Microbiology,

Rama medical College, Hospital and Research

Centre, Kanpur, UP- 209217

E-mail: homsharan@gmail.com, saggimishra@rediffmail.com

Ph: $00918005384934,00918738902326,00919839188620$

ABSTRACT: BACKGROUND: Surgical site infection is the second most common nosocomial infection after urinary tract infection and contributes to a significant percentage of morbidity and mortality in patients. OBJECTIVES: The objective was to find out SSI rate and determining the factors which are influencing the infection rate. METHODS: A total of 150 samples from surgical site were collected and bacterial isolates identified by standard methods. Antibiotic susceptibility testing was performed by Kirby-Bauer disc diffusion method. RESULTS: Most common bacteria isolated from surgical site infection was Staphylococcus aureus (31.58\%) followed by Klebsiella pneumoniae (26.31\%), Pseudomonas aeruginosa (15.79\%), E.coli (10.53\%), Acinetobacter (10.53\%) and Proteus mirabilis (5.26\%). Percentage of MRSA, ESBL production in E.coli and Klebsiella pneumoniae were 33.33\%, 50\% and 60\% respectively. All the strains of Staphylococcus aureus were sensitive to Vancomycin. Most of the strains of gram negative bacilli were sensitive to Amikacin. CONCLUSION: Surgical site infection prolong the hospital stay, increases the treatment cost, bed occupancy in ward and patient morbidity. Rapid and accurate detection of these pathogens and their antibiotic susceptibility pattern is important for prompt treatment, can prevent the emergence and dissemination of drug resistance. A little modification of determinants can reduce the SSI rate in a hospital to a costeffective way.

KEYWORDS: Surgical Site infection; Methicillin resistant Staphylococcus aureus; Extended spectrum $\beta$-lactamase; Determinants.

INTRODUCTION: One of the major problems faced by the surgeons these days is to deal with surgical site infection as most of them are caused by multi drug resistant bacteria ${ }^{1}$.

Despite improvement in operating room practices, instrument sterilization methods, better surgical techniques and the best efforts of infection prevention strategies, surgical site infections remain a major cause of hospital-acquired infections and rates are increasing globally even in hospitals with modern facilities and standard protocols of preoperative preparation and antibiotic prophylaxis. Moreover, in developing countries where resources are limited, even basic life-saving operations, such as appendicectomies and caesarean sections, are associated with high infection rates and mortality². 
The present study was undertaken to find out surgical site infection rate, to determining the antibiotic susceptibility pattern of isolated aerobic bacteria and the factors which are influencing the infection rate.

MATERIALS AND METHODS: A total of 150 samples from surgical site in General Surgery, Obstetric-Gynaecology, Orthopaedic, ENT and Ophthalmology Departments, which were clean, clean-contaminated and suspected of surgical site infection submitted to the Microbiology Laboratory of Rama Medical College, Hospital and Research Centre, Kanpur included in the study.

INCLUSION CRITERIA: Only those swabs from surgical site which were clean, clean contaminated and suspected of surgical site infection were included.

EXCLUSION CRITERIA: Surgical site wounds formed from cases directly related to skin, subcutaneous tissue, abscesses \& infected sebaceous cyst, etc. were excluded.

SAMPLE COLLECTION AND TRANSPORTATION: The discharge was collected after taking an informed written consent with two sterile swabs using aseptic precautions before dressing of wounds in the morning. The discharge collected swabs were transported to Microbiology Laboratory within 30 minutes for further processing.

CULTURE METHODS: One swab was used for making a smear \& other swab inoculated onto blood agar \& mac conkey agar and incubated at $37^{\circ} \mathrm{C}$ for 24 hours in $7-10 \% \mathrm{Co}_{2}$ concentration. The isolated organisms were identified by standard microbiological techniques ${ }^{3}$. All the isolates were tested for antimicrobial susceptibility by Kirby-Bauer disk diffusion method on MuellerHinton agar. MRSA and ESBL were detected by CLSI guidelines ${ }^{4}$.

\section{QUALITY CONTROL}

1. Staphylococcus aureus ATCC 25923-0xacillin susceptible.

2. Staphylococcus aureus ATCC 43300-Oxacillin resistant.

3. Klebsiella pneumoniae ATCC 700603- ESBL positive.

4. Escherichia coli ATCC J53RI(TEM ESBL)- ESBL positive.

RESULTS: Among 150 samples, bacteriologically proven surgical site infection was identified in 19 patients. Therefore, the prevalence of culture positive surgical site infection was $12.67 \%$.

DISCUSSION: Surgical site infection rate as reported by different workers varies from as low as $2.8 \%$ to as high as $49.5 \% 5,6$ (table 9). The high rate of Surgical site infection was reported by some authors $6,7,10$ which may be due to inclusion of all types of wounds. The lower rate of surgical site infection was reported by some authors ${ }^{5,12}$, may be due to differences in working conditions and hospital setup.

The most common bacteria which causes SSI was Staphylococcus aureus followed by Klebsiella pneumoniae, Pseudomonas aeruginosa, E.coli, Acinetobacter \& Proteus mirabilis (Table 1).The organisms which causes SSIs change from place to place and from time to time even in the same place. 
The incidence of MRSA was $33.33 \%$ (Table-2).All the strains of MRSA were sensitive to vancomycin, which could have relevant clinical use in the antibiotic policy guidelines for hospital. ESBL production was detected in $50 \%$ strains of E.coli \& $60 \%$ of Klebsiella pneumoniae (Table-3). With the spread of ESBL strains in hospital, co-resistance found to aminoglycosides, fluoroquinolones and tetracycline indicating multidrug resistance pattern. A therapeutic alternative has been recommended to prevent drug resistance among other organisms and hence, there is a need to formulate an antibiotic policy. High degree of resistance was observed against ciprofloxacin, ampicillin, gentamicin \& tetracycline but most sensitive drugs were amikacin \& Imipenem (Table-2).

It is seen from the above study that most of the bacteria were resistant to commonly used antibiotics due to overuse of antibiotics resulting in selection of resistant strains. It is necessary to know the sensitivity of different bacteria in surgical site infection for two reasons; firstly, to select the appropriate antibiotics to avoid the emergence or overgrowth of resistant bacteria to currently used antimicrobial agents and secondly, these resistant bacteria can cause cross infection to other patients.

Surgical site infection rate is increased in preoperative hospital stay due to severity of illness and comorbid conditions requiring therapy before operation ${ }^{16}$. Preoperative hospital stay also promotes acquisition of multidrug resistant hospital strains ${ }^{17,18,19}$.

In our study, surgical site infection rate was more in emergency operation than elective one, which may be due to sub-optimal preoperative antibiotic prophylaxis, suboptimal preoperative preparation and emergency operations were more likely to be dirty.

Duration of operation explained by decrease resistance due to increase blood loss and surgical trauma due to operative instruments which increase bacterial contamination.

The pre-existing illness increase the risk of surgical site infection due to increase in preoperative hospital stay which favour the bacterial colonization and increase the infection rate.

CONCLUSION: Proper infection control measures and a sound antibiotic policy should reduce SSIs in the future. A little modification in these determinants can reduce the SSIs rate in a hospital to a cost-effective way.

CONFLICTING INTERESTS: The authors have no conflicting interests.

\section{REFERENCES:}

1. Bergogne BE, Dearce D and Joly MC.Opportunistic nosocomial multiple resistant bacterialinfections, their treatment and prevention. Journal of Antimicrobial Chemotherapy, 1993; 32: Suppl A: 39-47.

2. Mawalla B,Mshana SE,Chalya PL,Imirzalioglu C,Mathalu W. Predictors of surgical site infections among patients undergoing major surgery at Bugando medical centre in northwestern Tanzania.BMC Surg.2011 Aug 31;11:21.

3. Collee JG, Miles RS, Watt B. Tests for the identificatin of bacteria .In:collee JG, Marmion BP, Fraser AG, Simmons A,editors. Mackie and Mc Cartney Practical Medical Microbiology.14th ed. London: Churchill Livingstone;2006:131-149.

4. Performance Standards for Antimicrobial Susceptibility Testing; Twenty- First Informational Supplement .CLSI document M100 S21. Wayne, PA: Clinical and Laboratory Standards Institute;2011. 
5. Olson M, O'Connor M, Schwartz ML. Surgical wound infections. A 5-year prospective study of 20,193 wounds at the Minneapolis VA Medical Center..Ann Surg 1984 Mar; 199(3): 253-259.

6. Agarwal PK, Agarwal M, Bal A, Gahlaut YVS. Incidence of postoperative wound infection at Aligarh. Indian J Surg 1984 Jun; 46: 326-333.

7. DeSa LA, Sathe MJ, Bapat RD. Factors influencing wound infection (A prospective study of 280 cases). J Postgrad Med 1984 Oct; 30(4): 232-236.

8. Murthy R, Sengupta S, Maya N, Shivananda PG. Incidence of postoperative wound infection and their antibiogram in a teaching and referral hospital. Indian J Med Sci 1998 Dec; 52(12): 553-555.

9. Anvikar AR, Deshmukh AB, Karyakarte RP, Damle AS, Patwardhan NS, Malik AK et al. A one year prospective study of 3280 surgical wounds. Indian J Med Microbiol 1999; 17(3)L 129-132.

10. Eriksen HM, Chugulu S, Kondo S, Lingaas E. Surgical site infections at Kilimanjaro Christian Medical Center. J Hosp Infect 2003 Sept; 55(1): 14-20.

11. Lilani SP, Jangale N, Chowdhary A, Daver GB. Surgical site infection in clean and cleancontaminated cases. Indian J Med Microbiol 2005; 23(4): 249-252.

12. Shojaei H, Borjian S, Shooshtari PJ, Shirani S. Surveillance of clean surgical procedures: Anindicator to establish a baseline of a hospital infection problem in a developing country, Iran. Indian J Surg 2006; 68: 89-92.

13. Chattopadhyay R, Zaroukian S, Potvin E. Surgical site infection rates at the Pontiac Health care Centre, a rural community hospital. Can J Rural Med 2006 Winter; 11 (1): 41-48.

14. Giri BR, Pant HP, Shankar PR, Sreeramareddy CT, Sen PK. Surgical site infection and antibiotics use pattern in a tertiary care hospital in Nepal. J Pak Med Assoc 2008 Mar; 58(3): 148-151.

15. Sangrasi AK, Leghari AA, Memon A, Talpur AK, Qureshi GA, Memon JM. Surgical siteinfection rate and associated risk factors in elective general surgery at a public sector medical university in Pakistan. Int Wound J 2008 Mar; 5(1): 74- 78.

16. Mangram AJ, Horan TC, Pearson ML, Silver LC, Jarvis WR. The Hospital Infection Control Practices Advisory Committee. Guideline for Prevention of Surgical Site Infection 1999. Infect Control Hosp Epidemiol 1999; 20: 247-280.

17. Yalcin AN and Bakir M. Postoperative wound infections. Journal of Hospital Infection 1995; Apr 29(4): 305-309.

18. Jacob DT, Richard L and Simmons. Preoperative antimicrobial prophylaxis. Master of surgery. 1998; 3rd edition, Vol.1; 134-145.

19. Shanson DC. Hospital Infection. Microbiology in clinical practice 1999; 3rd Edition: 429-458. 
Table 1:Aerobic bacteria isolated from surgical site infection

\begin{tabular}{|l|c|c|}
\hline Name of bacteria & No. of isolates & Percentage \\
\hline Staphylococcus aureus & 6 & 31.58 \\
\hline Klebsiella pneumoniae & 5 & 26.31 \\
\hline Pseudomonas aeruginosa & 3 & 15.79 \\
\hline Escherichia coli & 2 & 10.53 \\
\hline Acinetobacter & 2 & 10.53 \\
\hline Proteus mirabilis & 1 & 5.26 \\
\hline Total & 19 & 100.00 \\
\hline
\end{tabular}

Table 2: Antibiotic susceptibility pattern of isolates

\begin{tabular}{|c|c|c|c|c|c|c|c|}
\hline Antibiotics & $\begin{array}{l}\text { Disc } \\
\text { Conc. } \\
\mu \mathrm{g}\end{array}$ & $\begin{array}{l}\text { S. } \\
\text { aureus } \\
\text { n.6 }\end{array}$ & $\begin{array}{l}\text { K.pneum } \\
- \\
\text { - oniae } \\
\text { n.5 }\end{array}$ & $\begin{array}{l}\text { P.aeru- } \\
\text {-ginosa } \\
\text { n.3 }\end{array}$ & $\begin{array}{l}\text { E. } \\
\text { coli } \\
\text { n.2 }\end{array}$ & \begin{tabular}{|l|} 
Acinet \\
o- \\
- \\
bactor \\
n.2 \\
\end{tabular} & \begin{tabular}{|l|} 
p. \\
mirabili \\
s \\
n.1
\end{tabular} \\
\hline Penicillin & $\begin{array}{l}10 \\
\text { units }\end{array}$ & Nil & NT & NT & NT & NT & NT \\
\hline Gentamicin & 10 & $1(16.67 \%)$ & $2(40 \%)$ & $2(66.67 \%)$ & $1(50 \%)$ & $\begin{array}{l}1(50 \% \\
)\end{array}$ & Nil \\
\hline $\begin{array}{l}\text { Cotrimoxazol } \\
\text { e }\end{array}$ & $\begin{array}{l}23.75 / \\
1.25\end{array}$ & $2(33.33 \%)$ & NT & NT & NT & NT & NT \\
\hline Tetracycline & 30 & $1(16.67 \%)$ & Nil & NT & Nil & NT & Nil \\
\hline Erythromycin & 15 & $2(33.33 \%)$ & NT & NT & NT & NT & NT \\
\hline Vancomycin & 30 & $6(100 \%)$ & NT & NT & NT & NT & NT \\
\hline Cefoxitin & 30 & $4(66.67 \%)$ & NT & NT & NT & NT & NT \\
\hline Ampicillin & 10 & NT & Nil & $\mathrm{Nil}$ & $\mathrm{Nil}$ & Nil & Nil \\
\hline Amikacin & 30 & $\begin{array}{l}5 \\
(88.33 \%)\end{array}$ & $\begin{array}{l}4 \\
(80 \%)\end{array}$ & $\begin{array}{l}3 \\
(100 \%)\end{array}$ & $\begin{array}{l}2 \\
(100 \%)\end{array}$ & $\begin{array}{l}1 \\
(50 \%)\end{array}$ & \begin{tabular}{|l|}
1 \\
$(100 \%)$
\end{tabular} \\
\hline Cefotaxime & 30 & NT & $2(40 \%)$ & $2(66.67 \%)$ & $1(50 \%)$ & $\begin{array}{l}1(50 \% \\
)\end{array}$ & $1(100 \%)$ \\
\hline $\begin{array}{l}\text { Piperacillin- } \\
\text { Tazobactam }\end{array}$ & $\begin{array}{l}100 / 1 \\
0 \\
\end{array}$ & NT & NT & $2(66.67 \%)$ & NT & $\begin{array}{l}1(50 \% \\
)\end{array}$ & NT \\
\hline Ciprofloxacin & 5 & Nil & Nil & Nil & Nil & Nil & Nil \\
\hline Polymyxin B & $\begin{array}{l}300 \\
\text { Units }\end{array}$ & NT & NT & $2(66.67 \%)$ & NT & NT & NT \\
\hline Imipenem & 10 & NT & $\begin{array}{l}5 \\
(100 \%)\end{array}$ & $\begin{array}{l}3 \\
(100 \%)\end{array}$ & $\begin{array}{l}2 \\
(100 \%)\end{array}$ & \begin{tabular}{|l|}
2 \\
$(100 \%$ \\
)
\end{tabular} & $\begin{array}{l}1 \\
(100 \%)\end{array}$ \\
\hline
\end{tabular}

Abbreviations:NT-Not tested. 
Table 3: ESBL production rate in gram negative bacilli of surgical site infection

\begin{tabular}{|l|c|l|c|}
\hline Name of bacteria & $\begin{array}{l}\text { Number of } \\
\text { isolates }\end{array}$ & $\begin{array}{l}\text { No. } \\
\text { isolate } \\
\text { producing } \\
\text { ESBL }\end{array}$ & $\begin{array}{l}\text { Percentage } \\
\text { of ESBL }\end{array}$ \\
\hline Klebsiella pneumoniae & 5 & 3 & 60.00 \\
\hline E.coli & 2 & 1 & 50.00 \\
\hline Total & 7 & 4 & 57.14 \\
\hline
\end{tabular}

Table 4: Surgical site infection rate in pre-operative hospital stay

\begin{tabular}{|l|c|c|c|}
\hline $\begin{array}{l}\text { Preoperative hospital } \\
\text { stay (days) }\end{array}$ & $\begin{array}{l}\text { Total No. of } \\
\text { cases }\end{array}$ & $\begin{array}{l}\text { No. of } \\
\text { infected } \\
\text { cases }\end{array}$ & Percentage \\
\hline $1-7$ & 105 & 8 & 7.62 \\
\hline $8-14$ & 28 & 6 & 21.43 \\
\hline $15-21$ & 11 & 3 & 27.27 \\
\hline Above 21 & 6 & 2 & 33.33 \\
\hline Total & 150 & 19 & 12.67 \\
\hline
\end{tabular}

Table 5:Surgical site infection rate in patients receiving preoperative antibiotic prophylaxis

\begin{tabular}{|l|l|l|c|}
\hline $\begin{array}{l}\text { Preoperative antibiotic } \\
\text { therapy }\end{array}$ & $\begin{array}{l}\text { Total No. of } \\
\text { cases }\end{array}$ & $\begin{array}{l}\text { No. of } \\
\text { infected } \\
\text { cases }\end{array}$ & Percentage \\
\hline Received & 68 & 4 & 5.88 \\
\hline Not received & 82 & 15 & 18.29 \\
\hline Total & 150 & 19 & 12.67 \\
\hline
\end{tabular}

Table 6: Surgical site infection and nature of surgery

\begin{tabular}{|l|c|c|c|}
\hline Type of operation & $\begin{array}{l}\text { Total No. of } \\
\text { cases }\end{array}$ & $\begin{array}{l}\text { No. of infected } \\
\text { cases }\end{array}$ & Percentage \\
\hline Emergency operations & 36 & 7 & 19.44 \\
\hline Elective operations & 114 & 12 & 10.53 \\
\hline Total & 150 & 19 & 12.67 \\
\hline
\end{tabular}


Table 7:Surgical site infection and duration of operation

\begin{tabular}{|l|c|c|c|}
\hline Duration of operation & $\begin{array}{l}\text { Total No. of } \\
\text { cases }\end{array}$ & $\begin{array}{l}\text { No. } \\
\text { infected } \\
\text { cases }\end{array}$ & Percentage \\
\hline Less than 30 min & 19 & Nil & Nil \\
\hline 30 min to 1 hour & 46 & 3 & 6.52 \\
\hline More than 1 hour & 85 & 16 & 18.82 \\
\hline Total & 150 & 19 & 12.67 \\
\hline
\end{tabular}

Table 8: Surgical site infection rate in pre-existing illness

\begin{tabular}{|l|c|c|c|}
\hline Pre-existing illness & $\begin{array}{l}\text { Total No. of } \\
\text { cases }\end{array}$ & $\begin{array}{l}\text { No. of } \\
\text { infected } \\
\text { cases }\end{array}$ & Percentage \\
\hline Diabetes mellitus & 22 & 5 & 22.73 \\
\hline Malignancy & 20 & 1 & 5 \\
\hline Other illness & 7 & 1 & 14.29 \\
\hline Total & 49 & 7 & 14.29 \\
\hline
\end{tabular}

Table 9: Surgical site infection rate in different studies

\begin{tabular}{|c|c|c|c|}
\hline Study & Year & Country & $\begin{array}{l}\text { Surgical site } \\
\text { infection rate } \\
(\%)\end{array}$ \\
\hline Olson $\mathrm{M}$ et $\mathrm{al}^{5}$ & 1984 & Minneapolis, US & 2.80 \\
\hline Agarwal PK et al ${ }^{6}$ & 1984 & Aligarh, India & 49.50 \\
\hline Desa LA et $\mathrm{al}^{7}$ & 1984 & Mumbai, India & 18.92 \\
\hline Murthy $\mathrm{R}$ et $\mathrm{al}^{8}$ & 1998 & Manipal, India & 12.00 \\
\hline Anvikar AR et al ${ }^{9}$ & 1999 & Aurangabad, India & 6.09 \\
\hline Eriksen HM et al ${ }^{10}$ & 2003 & Tanzania & 19.40 \\
\hline Lilani SP et al ${ }^{11}$ & 2005 & Mumbai, India & 8.95 \\
\hline Shojaei H et al ${ }^{12}$ & 2006 & Iran & 4.9 \\
\hline Chattopadhyay R et al ${ }^{13}$ & 2006 & Canada & 5.54 \\
\hline Giri BR et al ${ }^{14}$ & 2008 & Nepal & 7.3 \\
\hline Sangrasi AK et al ${ }^{15}$ & 2008 & Pakistan & 13.0 \\
\hline Present study & 2012 & Kanpur, India & 12.67 \\
\hline
\end{tabular}




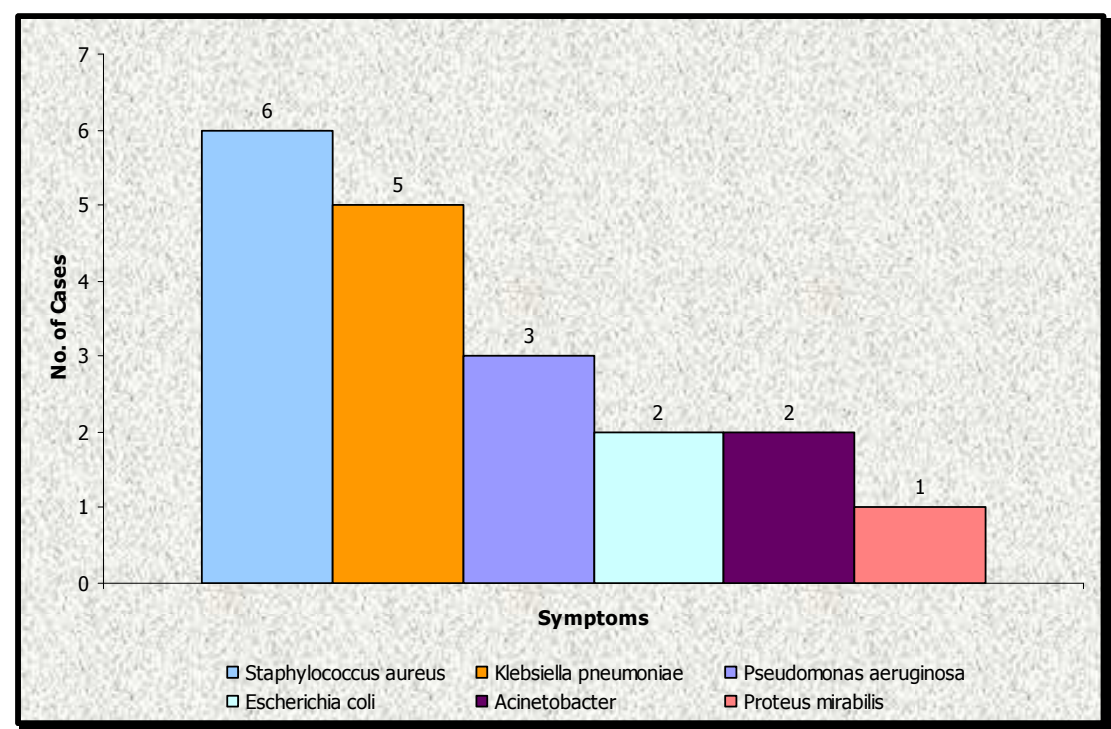

Figure 1: Aerobic bacteria isolated from surgical site infection

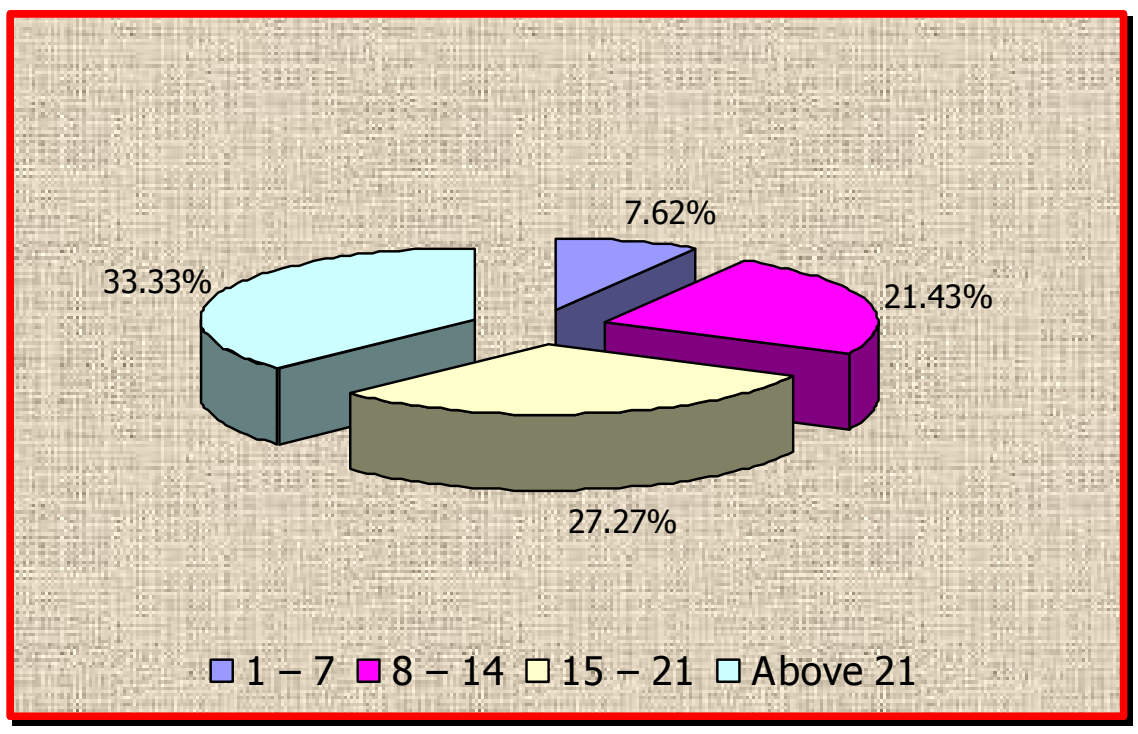

Figure 2: Surgical site infection rate in pre-operative hospital stay 